\title{
Kraft, Arbeit und Maschinen: Ökomarxistische Perspektiven in der sozialwissenschaftlichen Klimaforschung
}

\author{
Daniela Russ
}

Erscheint in: Schlüsselwerke der sozialwissenschaftlichen Klimaforschung. Bielefeld: transcript.

Galt Marx lange als wenig relevant für die sozialwissenschaftliche Umweltund Klimaforschung, so sind in den letzten Jahrzehnten verschiedene ökomarxistische Stränge entstanden, die sein Werk für dieses Feld erschließen. Neben der Auseinandersetzung mit Marx“ ökologischen Gedanken und dem Begriff des Metabolismus (Foster 1999, Burkett 1999, $\rightarrow$ Grundmann), ist dabei auch eine neue Auseinandersetzung mit der Geschichte der Produktivkräfte entstanden, in der es um die Aneignung und Nutzung fossiler Brennstoffe und ihr Verhältnis zu menschlicher Arbeit geht. Ziel dieser Arbeiten ist es, die Entstehung und Bedeutung unserer gegenwärtigen Wirtschaft, in der wir nicht leben können, ohne am Ausstoß von Treibhausgasen zu partizipieren, besser zu begreifen. Kurz: Wie verhält sich die kapitalistische Produktionsweise zur Ausbreitung der fossilen Brennstoffnutzung?

Besonders relevant für diese Strömung des Ökomarxismus ist Marx ‘ Kapitel $\mathrm{zu}$ „Maschinerie und große Industrie“ im ersten Band des Kapitals (Marx 1968). Im Zusammenhang des Gesamtwerkes geht es Marx hier um die Produktion von relativem Mehrwert, das heißt um den zusätzlichen Gewinn, den Fabrikbesitzer machen können, indem sie die Produktivität steigern. Marx beschreibt in diesem Kapitel die Technisierung der Produktion, wie sie zwischen 1750-1850 in Großbritannien stattfand, als Übergang von der Maschine zum Maschinensystem - einer aufeinander abgestimmten Apparatur aus Bewegungsmaschine, Transmissionsmechanismus und Arbeitsmaschine (393). Bei der Bewegungsmaschine handelt es sich um die Antriebskraft des Produktionsapparates, wobei Marx hier zunächst keinen Unterschied macht zwischen der Nutzung menschlicher oder tierischer 
Kräfte, der Naturkraft des Wassers oder der Kohle. Über einen Transmissionsmechanismus - Schwungräder, Treibwellen, allerhand Schnüre und Geschirr - wird die Kraft auf die Arbeitsmaschine übertragen, die die eigentliche Veränderung des Arbeitsgegenstandes vollführt. Die Arbeiter arbeiten der Maschine nur zu, sie sind ,Anhängsel' der Maschine. Im Unterschied zur Manufaktur, die von den einzelnen handwerklichen Schritten aus entworfen war, ist die Produktion im Maschinensystem objektiv, wissenschaftlich organisiert: die Arbeiterin findet den gesamten Apparat als fertige Produktionsbedingung vor (407).

Aus dieser Produktionsweise ergeben sich Produktionsverhältnisse, das heißt neue und veränderte soziale Beziehungen. Für den Ökomarxismus sind zwei von Marx' Beobachtungen besonders relevant: Zwar entwertet diese Art der Produktion die handwerkliche Ausbildung und senkt die Arbeitslöhne, da viele der Arbeiten an der Maschinerie auch von Frauen und Kindern geleistet werden können (416 f.). Gleichzeitig liegt in dieser Art zu produzieren, in die sich die Arbeiterinnen gemeinsam fügen müssen, jedoch schon die Möglichkeit ihres gemeinsamen politischen Kampfes - gemeinsam können sie die Produktion auch blockieren. Zweitens führt die rationale Organisation des Maschinensystems dazu, dass Menschen und Tiere letztendlich als Antriebskräfte ersetzt werden, da sie zu widerspenstig sind, ein „unvollkommenes Produktionsinstrument gleichförmiger und kontinuierlicher Bewegung“ (396). Insbesondere die Dampfmaschine, die „,hre Bewegungskraft selbst erzeugt aus der Verspeisung von Kohle und Wasser, dessen Kraftpotenz ganz unter menschlicher Kontrolle steht," befreit die Industrie von räumlichen Schranken und erlaubt ihre Konzentration in den Städten, ,statt sie wie das Wasserrad über das Land zu verteilen“ (398).

Obwohl Marx sehr wohl eine Vorstellung ökologischer Grenzen hatte (Saito 2016), konnte er nicht wissen, als wie bedeutsam diese Umstellung von Wasser- auf Dampfkraft sich herausstellen würde. Was bei Marx nur eine Folge des Maschinensystems ist, wird in Andreas Malms Fossil Capital (2016) zur zentralen Frage: Warum beginnen die Besitzer von Textilfabriken in Großbritannien Anfang des 19. Jahrhunderts auf Dampfkraft umzustellen (57)? Zu dieser Zeit gab es die Dampfmaschine schon ein ganzes Jahrhundert und auch gute Flusslagen waren noch zu haben (Kap. 4-5). Malm zeigt, dass es entgegen der Behauptung einiger Historiker (Sieferle 1982, Wrigley 2010) nicht der Holz- oder Wassermangel war, der die Fabrikbesitzer zur 
Dampfkraft trieb. Auf dem Land, wo die Textilbetriebe zum überwiegenden Teil angesiedelt waren, war nicht die Energie knapp, sondern die Arbeitskraft, die sich disziplinieren ließ. Malm bestätigt Marx ‘ Beobachtung, dass die Dampfkraft die industrielle Produktion von den geografischen Gegebenheiten unabhängiger macht und es den Fabrikbesitzern - jetzt fossile Kapitalisten - ermöglicht, auf die billigere, diszipliniertere Arbeiterschaft in den Städten zurückzugreifen (160). Darüber hinaus unterliegt die Dampfkraft im Unterschied zur Wasserkraft keinen saisonalen und wetterbedingten Schwankungen: sie erlaubt eine volle Auslastung des Kapitals (192 f.).

Mit der Entstehung und Ausbreitung einer Produktionsweise, die auf fossilen Brennstoffen basiert, entsteht auch eine Gruppe von Arbeitern - Bergleute, Heizer, Eisenbahner, Schiffer - die den ständigen Nachschub dieser Stoffe gewährleistet. Wie Timothy Mitchell in Carbon Democracy (2011) zeigt spielen diese Energie-Arbeiter in den Arbeiterbewegungen der westlichen Industriestaaten des 19. und frühen 20. Jahrhunderts eine entscheidende Rolle. Die Kohle bewegt sich über enge, speziell dafür eingerichtete Bahnen durch die Gesellschaft, die von Arbeiterinnen unterhalten werden. Die gesellschaftliche Produktion und Verteilung großer Energiemengen liegt nun in den Händen einer kleinen Gruppe von Arbeiterinnen. In Streiks und Sabotagen setzten sie ihre Fähigkeit, die Brennstoffflüsse zu unterbrechen und die gesamte Wirtschaft lahmzulegen, dazu ein, politische Macht zu gewinnen. In den Jahrzehnten vor dem ersten Weltkrieg erkämpfen sie so nicht nur das Wahlrecht, sondern auch das Recht, sich zu organisieren und zu streiken (19-27). Die modernen Massendemokratien sind wesentlich aus diesen Kämpfen entstanden, und noch heute sind unter den mächtigsten Gewerkschaften viele, die am Brennstofffluss gediehen sind.

Die Materialität der Ressourcen und die Struktur fossiler Infrastrukturen sind zentral, wenn es darum geht, ob Arbeiterinnen die Energieströme der Gesellschaft blockieren und als politische Waffe einsetzen können. Die politische Macht von Arbeitern und die Durchsetzung ihrer demokratischen Forderungen erfordert die Kontrolle einer Maschine (83). Im Unterschied zur Kohle, die unter erheblichem Aufwand und großer Gefahr aus dem Berg herausgehämmert werden muss, strömt das Erdöl selbst an die Oberfläche, sobald es einmal angezapft ist. Und während die Kohleindustrie mit Eisenbahnen, Schiffslinien und Kanalgesellschaften verhandeln musste, um 
ihr Produkt zum Konsumenten zu bringen, kontrollierten die multinationalen Ölunternehmen - unter dem Schutz der USA und anderer westlicher Länder - sowohl die Pipelines als auch die Raffinerien. Die Zirkulation des Öls ließ sich mit deutlich weniger und stark verstreuten Arbeiterinnen gewährleisten als die der Kohle. Darüber hinaus hatten die Ölunternehmen häufig selbst ein Interesse daran, die Produktion zu drücken, um höhere Preise zu erzielen. In dieser Situation gelang es den Ölarbeiterinnen nicht in gleichem Maße, ihre Schlüsselpositionen in politische Repräsentation umzumünzen $(43,226)$.

Malms und Mitchells historische Studien verweisen auf einen engen Zusammenhang von fossiler Brennstoffnutzung und Kapitalismus, den Elmar Altvater bereits in ,The Social and Natural Environment of Fossil Capitalism“ (2007) herausgearbeitet hat: „fossil energy fulfils almost perfectly the requirements of the capitalist process of accumulation. It fits into capitalism's societal relation to nature." (41) Der Grund dafür liegt in der zeitlichen und räumlichen Unabhängigkeit von Produktion, die fossile Brennstoffe ermöglichen, sowie in ihrer flexiblen Anwendbarkeit, die längst die Marx'sche Bewegungsmaschine übersteigt. Malm diskutiert diesen Aspekt unter dem Begriff der zeitlichen und räumlichen Abstraktion (Kap. 7-8). In einer globalen Brennstoffwirtschaft ist die geografische Bedingtheit vieler Produktionsprozesse aufgehoben und Energie ist nur noch einer unter vielen anderen Faktoren - wie die Existenz günstiger Arbeitskraft - die über Investitionen entscheiden.

Das ökomarxistische Feld, in dem das Verhältnis von Arbeit, Kapital und Energie erforscht wird, ist erst in den letzten Jahren entstanden. Dementsprechend viele Fragen bleiben noch offen, von denen hier nur drei angesprochen werden sollen. Eine erste große Lücke dieser Forschungsrichtung ist die Rolle der Elektrifizierung, die um 1890 einsetzt und bald schon Dampf- und Wasserkraft in einem großtechnischen System vereint, das die Arbeiter in wenigen großen Kraftwerken konzentriert. Wie sinnvoll ist es, sich auf die fossilen Brennstoffe zu konzentrieren, wenn sich in modernen Energiesystemen fossile und nicht-fossile Quellen zu einem gewissen Grad komplementieren, das heißt gegenseitig erst ermöglichen? Müsste man nicht allgemeiner von Kapital an den energetischen Produktionsmitteln sprechen, gerade wenn es in Zukunft um eine vielleicht nicht-fossile, aber vermutlich noch immer kapitalistische organisierte Energiewirtschaft geht? 
Zweitens bleiben in der Geschichte fossilen Kapitals die vielen andere Nutzungsweisen der Naturstoffe unberücksichtigt: Kohle, Öl, und Gas wurden nicht nur als Brennstoff, sondern immer auch als Rohmaterial genutzt (die Kohle härtet das Eisen, sie bringt es nicht nur zum Schmelzen). Im zwanzigsten Jahrhundert entwickelte sich eine ganze chemische Industrie auf Grundlage der Kohlenwasserstoffe, so dass wir heute nicht nur abhängig sind von Benzin, sondern auch von Plastik. Diese Beziehungen innerhalb der Energiewirtschaft und zu anderen Sektoren hat der Ökomarxismus bisher noch nicht aufgegriffen, obwohl sie maßgeblich dafür waren und sind, wie profitabel die Förderung fossiler Brennstoffe ist.

Zuletzt bleibt - trotz Mitchells Arbeit - die Rolle des Staates in der Entwicklung und Verbreitung fossilen Kapitals noch unterbelichtet. Es sind weniger die Kohleminen, als die großen Trusts und Aktiengesellschaften der Öl- und Elektrizitätsindustrie, in denen sich Ende des neunzehnten Jahrhunderts das fossile Kapital zu konzentrieren beginnt. Wie Mitchell für die Ölindustrie zeigt, sind die Konzentration und die internationalen Geschäfte nur möglich durch die Regulierung und Unterstützung von Staaten. Spätestens im frühen zwanzigsten Jahrhundert ist der Ausbau der Energiewirtschaft dann ein explizit politisches Projekt der wirtschaftlichen Macht, Autonomie und Entwicklung.

Die ökomarxistische Forschung schreibt die Geschichte des fossilen Kapitals nicht als neutrale Chronik, sondern als Geschichte der Gegenwart. Sie stellt sich als Aufgabe, das Verhältnis von Arbeit und ,fossilem Kapital ${ }^{\text {‘ }}$ - den Eigentümern der Ressourcen und Infrastrukturen, die heute die Produktion aller Güter antreibt - als Gewordenes zu begreifen. Dabei beschreibt sie nicht nur, was gewesen ist, sondern zeigt auch auf, mit welchen historisch gewachsenen Subjektivitäten eine Politik heute rechnen muss, die Fragen von sozialer und ökologischer Gerechtigkeit zu verbinden versucht.

Im Unterschied $\mathrm{zu}$ vielen anderen Strömungen sozialwissenschaftlicher Klimaforschung hat die marxistische Tradition immer Natur und Gesellschaft in ihrer Verbundenheit gedacht: Dass die Gesellschaft in der Natur, mit natürlichen Stoffen und gegen ihren Widerstand, arbeitet, muss der Marxismus nicht erst wiederentdecken. Das ökomarxistische Denken 
entwickelt diese Einbindung der Arbeit in die Natur vor dem Hintergrund des Klimawandels weiter: Im fossilen Kapitalismus, in dem wir leben, geht jede Produktion und Konsumtion mit dem Ausstoß von Klimagasen einher. Das zentrale Dilemma besteht darin, dass fossile Produktionsmittel heute nicht nur die Mittel sind, die soziale Reproduktion ermöglichen, sondern auch diejenigen, die sie gefährden.

Daniela Russ

\section{Literaturverzeichnis}

Altvater, Elmar (2007): The Social and Natural Environment of Fossil Capitalism. In: Socialist Register 43, S. 37-49.

Burkett, Paul (1999): Marx and Nature: a Red and Green Perspective. New York: St. Martin's Press.

Foster, John Bellamy (1999): Marx's Theory of Metabolic Rift: Classical Foundations for Environmental Sociology. In: American Journal of Sociology 105 (2), S. 366-405.

Marx, Karl (1968): Maschinerie und große Industrie. In: Karl Marx Friedrich Engels - Werke, Band 23: Das Kapital, Band 1, Berlin: Dietz

Malm, Andreas (2016): Fossil Capital. The Rise of Steam Power and the Roots of Global Warming. London: Verso.

Mitchell, Timothy (2011): Carbon Democracy. Political Power in the Age of Oil. London: Verso.

Saito, Kohei (2016): Natur gegen Kapital: Marx“ Ökologie in seiner unvollendeten Kritik des Kapitalismus. Frankfurt: Campus.

Sieferle, Rolf Peter (1982): Der unterirdische Wald: Energiekrise und industrielle Revolution. München: C.H. Beck.

Wrigley, E. A. (2010): Energy and the English Industrial Revolution. Cambridge: Cambridge University Press. 